\title{
Insulin resistance, diabetes mellitus and thyroid dysfunction in patients with palmoplantar pustulosis: a case-controlled study
}

\author{
Hatice Ataş, Müzeyyen Gönül \\ Department of Dermatology, University of Health Sciences, Dışkapı Yıldırım Beyazıt Training and Research Hospital, Ankara, Turkey \\ Adv Dermatol Allergol 2017; XXXIV (3): 268-272 \\ DOI: $10.5114 / p d i a .2016 .61630$
}

\begin{abstract}
Introduction: Palmoplantar pustulosis (PPP) is a chronic pustular inflammatory skin disease; however, its pathogenesis is not well understood. Several factors, such as genetics, tobacco use and autoimmune issues, may contribute to this disease.

Aim: This research was conducted to investigate the relationships between insulin resistance, thyroid disease and PPP.

Material and methods: Thirty-three patients with PPP and 27 age- and gender-matched controls were analysed for their smoking histories, thyroid function tests, anti-thyroid peroxidase antibody (anti-TPO) levels, fasting glucose, fasting insulin levels and the homeostatic model assessment (HOMA) index for insulin resistance.

Results: We found significant differences between the PPP and control groups according to their tobacco use and anti-TPO levels ( $p=0.009$ and $p=0.009$, respectively). The proportion of tobacco use was $90 \%$ in the PPP patients and $63 \%$ in the controls. Gender and tobacco use were predictive risk factors for PPP in the multivariate analysis $(O R=141.7, p<0.0001$ and $O R=147.6, p=0.006$, respectively). An anti-TPO level $>35 \mathrm{U} / \mathrm{ml}$ and the presence of a thyroid abnormality were independent risk factors in the univariate, but not the multivariate analysis $(O R=4.2$, $p=0.025$ and $O R=5.4, p=0.004$, respectively). A moderate correlation between the gender and anti-TPO level was found ( $r=0.361, p=0.039$ ); however, the fasting glucose, insulin and HOMA index were not significant between the PPP and control groups.

Conclusions: Female gender and smoking were the most important risk factors for PPP; however, the increase in the anti-TPO level may be related to the predominance of females afflicted with this disease. Additional studies are necessary to clarify the relationships between PPP, thyroid disease and diabetes mellitus.
\end{abstract}

Key words: palmoplantar pustulosis, insulin resistance, diabetes mellitus, thyroid, contribution.

\section{Introduction}

Palmoplantar pustulosis (PPP) is a chronic pustular inflammatory skin disease. Its characteristics include chronically recurring sterile pustules frequently occurring with additional findings, such as yellow-brown macules (remnants of resolving pustules), erythema, scales and fissures on the palms of the hands and soles of the feet. It usually occurs in middle-aged women who smoke, and erupts repeatedly over months or years [1, 2]. Unfortunately, the pathogenesis of PPP is not well understood. Several factors may contribute to this disease, including genetics, tobacco use and autoimmune disorders [3, 4]. Additional studies are necessary to clarify the relationship between PPP and autoimmune disorders, such as thyroid disease, gluten sensitivity and type 2 diabetes mellitus $[3,5,6]$.

\section{Aim}

In this study, we aimed to investigate the possible relationships between PPP, insulin resistance and thyroid dysfunction.

\section{Material and methods}

This was a single centre, case-control study performed in the Department of Dermatology at the Diskapi Yildirim Beyazit Training and Research Hospital in 
Ankara, Turkey. Approval for this research was obtained from the Institutional Ethics Committee, and informed written consent was obtained from all of the subjects.

The controls were selected from the patients admitted to the dermatology clinic for nevi. The inclusion criteria for the patients with PPP were the following: a) recurring pustulosis on the feet or hands with a negative bacteriological study, b) no signs of psoriasis or eczema on the rest of the skin, and no personal history of psoriasis, c) diagnosed with PPP after clinical and/or histopathological investigations, d) no systemic treatment over the previous 3 months, including steroids, immunosuppressant agents, retinoids and phototherapy and e) no vascular events, chronic hepatic diseases, chronic renal diseases, malignancies, pregnancy, connective tissue disorders, Crohn's disease, coeliac disease, or any disease that could affect the immune system.

The patients were interviewed with regard to their a) past history of tonsillitis, autoimmune disease, thyroid disease and beginning of PPP symptoms, b) family history of PPP, diabetes and thyroid disease and c) tobacco use (classified as $\mathrm{A}=$ non-smoker (NS), B = smoker (S), B1 = smoker of 10 cigarettes or less/day and B2 = smoker of more than 10 cigarettes/day). All of the participants were analysed for their thyroid function, anti-thyroid peroxidase antibody (anti-TPO) levels, fasting glucose, fasting insulin levels and homeostatic model assessment (HOMA) index for insulin resistance (IR). Venous blood samples were taken from the subjects after 8 hours of fasting. The chemiluminescent immunometric assay method was used for the serum insulin (<12 mU/l), free T3 (FT3) (range: 2.3-4.2 pg/ $\mathrm{ml}$ ), free T4 (FT4) (range: $0.74-1.52 \mathrm{ng} / \mathrm{dl}$ ), anti-TPO $(<35 \mathrm{U} / \mathrm{ml})$ and anti-thyroglobulin (anti-TG) $(<40 \mathrm{U} / \mathrm{ml})$. Spectrophotometric methods were used to measure the thyroid-stimulating hormone (TSH) (0.55-4.78 mIU/l) and fasting blood glucose (70-100 mg/dl).

The HOMA index uses the formula described by Matthews et al. [7]: insulin $(\mathrm{mU} / \mathrm{l}) \times($ glucose $(\mathrm{mmol} / \mathrm{l}) / 22.5)$. The cut-off point to define IR was above $2.6 \mu \mathrm{U} / \mathrm{ml}$ in the HOMA.

\section{Statistical analysis}

The data were analysed using SPSS 15.0 software. The descriptive analyses were presented using the median, range and tables of the frequencies for the variables. A $\chi^{2}$ or Fisher's exact test was used for the discrete variables and the Student's $t$ test or Mann-Whitney $U$ test was used for the continuous variables, after detecting the normality according to the Kolmogorov-Smirnov test, which was used to compare the variables between the PPP and control groups, where appropriate. For the multivariate analysis, the possible factors (age, gender, smoking, family history of PPP, diabetes mellitus and thyroid disease, time of disease onset, duration of PPP, FT3, FT4, TSH, anti-TPO, anti-TG, fasting blood glucose, insulin levels and HOMA index) identified with the univariate analyses were further entered into the logistic regression analysis to determine the independent predictors of PPP. A $p$-value of less than 0.05 was considered to show a statistically significant result.

\section{Results}

This study included 33 patients with PPP (31 females, 2 males, median age of 40 years old) and 27 age- and gender-matched controls ( 23 females, 4 males, median age of 38 years old). The other demographic and laboratory characteristics of the subjects can be seen in Tables 1 and 2. The fasting glucose, insulin and HOMA index were not significant between the PPP patients and the controls ( $p=0.52, p=0.31$ and $p=0.22$, respectively). In addition, the IR according to the insulin and HOMA index was not found to be a significant risk factor for PPP $(p>0.05)$. The ratios of tobacco use were $90 \%$ in the PPP group and $63 \%$ in the control group, and there was a statistically significant difference between the groups $(p<0.009)$. A history of thyroid disease was found in $21 \%$ of the PPP patients, compared to $7 \%$ of the controls $(p=0.17)$. This rate was increased to $60 \%$ in the PPP group and $22 \%$ in the control group ( $p=0.003)$ when all of the thyroid abnormalities, including the history of thyroid disease and laboratory parameters, were evaluated. The median anti-TPO levels were $30 \mathrm{U} / \mathrm{ml}$ in the PPP group and $20 \mathrm{U} /$ $\mathrm{ml}$ in the control group. A significant difference was detected between the PPP and control groups according to the anti-TPO levels ( $p=0.009)$. If the anti-TPO level was $\geq 21 \mathrm{U} / \mathrm{ml}$, both the sensitivity and specificity of the antiTPO were $67 \%$. The median anti-TPO level was $34 \mathrm{U} / \mathrm{ml}$ in women with PPP and $11.5 \mathrm{U} / \mathrm{ml}$ in men with PPP. The autoantibody abnormality rate in patients with PPP was three-times higher than that in the control group (42\% vs. $15 \%, p=0.02$ ). The gender and tobacco use were predictive for PPP in the multivariate analysis $(O R=141.7$, $p<0.0001$ and $O R=147.6, p=0.006$, respectively). An anti-TPO $>35 \mathrm{U} / \mathrm{ml}$ and the presence of a thyroid abnormality were found to be independent risk factors in the univariate analysis $(O R=4.2, p=0.025$ and $O R=5.4$, $p=0.004$, respectively), but not in the multivariate analysis ( $p=0.39$ and $p=0.07$, respectively) (Table 3 ).

\section{Discussion}

The exact incidence and prevalence of PPP are not well known [8, 9]. Moreover, a determination of the prevalence of PPP was not possible according to our study, but we can say that it is a rare disease in our clinic.

The PPP is a pustular skin disorder that usually occurs in adults [2]. Although PPP is listed among the pustular variants of psoriasis in many textbooks, a recent genetic study has provided evidence that PPP may not be related to psoriasis [4]. In general, the disease is found in people between 20 and 60 years of age, which is compatible with 
Table 1. Comparison of some clinical features in palmoplantar pustulosis (PPP) and control groups

\begin{tabular}{|c|c|c|c|c|c|c|c|c|}
\hline \multirow[t]{2}{*}{ Parameter } & & \multicolumn{3}{|c|}{ PPP } & \multicolumn{3}{|c|}{ Controls } & \multirow[t]{2}{*}{$P$-value } \\
\hline & & $n(\%)$ & Median & Range & $n(\%)$ & Median & Range & \\
\hline \multicolumn{2}{|l|}{ Number of subjects } & 33 & & & 27 & & & \\
\hline \multicolumn{2}{|l|}{ Age [years] } & & 40 & $23-60$ & & 38 & $21-58$ & 0.55 \\
\hline \multirow[t]{2}{*}{ Gender } & Male & $2(6)$ & & & $4(14)$ & & & 0.39 \\
\hline & Female & $31(94)$ & & & $23(85)$ & & & \\
\hline \multirow[t]{2}{*}{ Tobacco use } & Smoker & $30(90)$ & & & $10(37)$ & & & 0.009 \\
\hline & Non-smoker & $3(9)$ & & & $17(63)$ & & & \\
\hline \multirow{2}{*}{$\begin{array}{l}\text { Family history of diabetes } \\
\text { mellitus }\end{array}$} & No & $15(45)$ & & & $16(60)$ & & & 0.29 \\
\hline & Yes & $18(55)$ & & & $11(40)$ & & & \\
\hline \multirow{2}{*}{$\begin{array}{l}\text { Family history of thyroid } \\
\text { disease }\end{array}$} & No & $24(73)$ & & & $19(70)$ & & & 0.53 \\
\hline & Yes & $9(27)$ & & & $8(30)$ & & & \\
\hline \multirow{2}{*}{$\begin{array}{l}\text { Medical history of thyroid } \\
\text { disease }\end{array}$} & Yes & $7(21)$ & & & $2(7)$ & & & 0.17 \\
\hline & No & $26(79)$ & & & $25(93)$ & & & \\
\hline \multicolumn{2}{|c|}{ Disease duration time [years] } & & 4 & $1-20$ & & & & \\
\hline \multicolumn{2}{|l|}{ Smoking time [years] } & & 20 & $0-40$ & & & & \\
\hline
\end{tabular}

Table 2. Comparison of some laboratory results in patients with palmoplantar pustulosis (PPP) and the control group

\begin{tabular}{lccccc}
\hline \multirow{2}{*}{ Parameter } & \multicolumn{2}{c}{ PPP } & \multicolumn{2}{c}{ Controls } & \multirow{2}{*}{-value } \\
\cline { 2 - 5 } & Median & Range & Median & Range & 0.52 \\
\hline Fasting blood glucose [mg/dl] & 92 & $71-286$ & 88 & $70-210$ & 0.31 \\
\hline Insulin [mU/l] & 9 & $3-27$ & 8 & $3-20$ & 0.22 \\
\hline HOMA index & 2.1 & $0.8-11.8$ & 1.8 & $0.6-6.9$ & 0.73 \\
\hline FT3 [pg/ml] & 3 & $2.1-7$ & 3.1 & $2.3-4.2$ & 0.48 \\
\hline FT4 [ng/dl] & 1.2 & $0.3-9.4$ & 1.1 & $0.08-3.7$ & 0.42 \\
\hline TSH [mlU/l] & 2.4 & $0.03-12$ & 3 & $0.01-6.7$ & 0.17 \\
\hline Anti-TG [U/ml] & 25 & $2-1000$ & 20 & $5-300$ & 0.009 \\
\hline Anti-TPO [U/ml] & 30 & $2-1500$ & 20 & $3-1500$ & \\
\hline F-fres & & &
\end{tabular}

F-free, PPP-palmoplantar pustulosis, TG - thyroglobuline, TPO - thyroid peroxidase.

Table 3. Effective parameters of palmoplantar pustulosis in uni/multivariate analysis

\begin{tabular}{lccccccc}
\hline \multirow{2}{*}{ Predictors } & \multirow{2}{*}{ Category } & \multicolumn{3}{c}{ Univariate analysis } & \multicolumn{3}{c}{ Multivariate analysis } \\
\cline { 3 - 9 } & & $O R$ & $95 \% \mathrm{Cl}$ & $\boldsymbol{P}$-value & OR & $95 \% \mathrm{Cl}$ & $P$-value \\
\hline Anti-TPO & High/normal & 4.2 & $1.2-15$ & $\mathbf{0 . 0 2 5}$ & 0.9 & $0.9-1.1$ & 0.39 \\
\hline Presence of any thyroid abnormality & Yes/no & 5.4 & $1.7-16.9$ & $\mathbf{0 . 0 0 4}$ & 14.6 & $0.8-273$ & $\mathbf{0 . 0 7}$ \\
\hline Tobacco use & S/NS & 17.1 & $4.1-70$ & $<0.0001$ & 148 & $4.2-5141$ & 0.006 \\
\hline Gender & F/M & 89.1 & $15.1-529$ & $<0.0001$ & 142 & $8.9-2258$ & $<0.0001$ \\
\hline
\end{tabular}

$\mathrm{Cl}$ - confidence interval, ${ }^{\S} \mathrm{Normal}<35 \mathrm{U} / \mathrm{ml}$, NS - non-smoker, OR - odds ratio, TPO - thyroid peroxidase, F-female, M-male

our patients' age range (23-60 years old) [10]. Women and smokers are more frequently affected [1, 2]. According to gender in PPP patients, an 8.8-fold increased risk was found in the females when compared to the males in the multivariate analysis $(O R=141.7, p<0.0001)$ in this study.
Tobacco use plays a role in the pathogenesis of PPP; however, the prevalence of tobacco use in patients with PPP is different based on different studies (range: 42$100 \%)[9,11]$. In this study, the ratio was $90 \%$ in 33 patients with PPP. When we compared it with the rate of 
the control group, the difference was found to be highly significant $(90 \%$ vs. $63 \%, p<0.009)$. Smoking was a $32.7-$ fold increased risk factor for PPP in the multivariate analysis $(H R=147.6, p=0.006)$.

The relationships between PPP and certain clinical features, such as gluten sensitivity, thyroid disease, diabetes mellitus and focal infections (like tonsillitis) have been demonstrated in recent studies $[5,6,12,13]$. The increased frequency of thyroid disorders varies based on different studies, while the prevalence of thyroid pathologies is about $5 \%$ in the general population [3]. The rate in the control group was $7 \%$ according to the history of thyroid disease in our study. In addition, high frequencies of thyroid disease and thyroid autoantibodies were found in the patients with PPP in one previous study [13]. In another study of PPP, 3 (25\%) and 2 (16\%) of 12 patients were diagnosed with thyroid diseases and antimicrosomal antibodies, respectively [3]. In these studies, the patients were compared with the general population, not with a control group; however, we used a case-control study.

The prevalence of thyroid pathologies in PPP ranged from $16 \%$ to $25 \%$ in some studies, and these rates are significantly higher than these found in the general population [3, 13-15]. Our rate was $21 \%$ according to the history of thyroid disease in the PPP group, which was three-times higher than in the control group (21\% vs. $7 \%$ ). Hashimoto's disease, multinodular goitre and hypothyroidism are the major associated thyroid disorders [3]. In this study, Hashimoto's disease was found in 4 patients, a multinodular goitre in one patient, Graves in 1 patient and subclinical hypothyroidism in one patient. When we considered all thyroid abnormalities, including thyroid function, TSH, T4, anti-TPO and anti-TG autoantibody abnormalities and a positive thyroid disease history, this rate was $60 \%$ in the PPP group and $22 \%$ in the control group. When we investigated the thyroid autoantibodies, the abnormality rate of the patients with PPP was approximately three times higher than in the controls ( $42 \%$ vs. $15 \%, p=0.02$ ). Thyroid antigen antibodies are more common in women, with a rate of $8.4 \%$ in the general population [16]. Gul et al. reported the frequency of thyroid autoantibodies in healthy Turkish individuals as 9.9\% [17]; however, this rate has been reported as $22.5 \%$ in women with PPP [13]. In this study, a moderate correlation between the gender and anti-TPO levels was found $(r=0.361, p=0.039)$, while the anti-TPO rate was threetimes higher in women than in men in the PPP group. However, in previous studies, the anti-TPO level was not been evaluated in a multivariate analysis. Moreover, the anti-TPO level was an independent risk factor in the univariate analysis, but not in the multivariate analysis in our study. High autoantibody levels can be related to the female gender because of the high tendency toward PPP in females.

Gimenez-Garcia et al. [3] demonstrated that all of their patients with PPP exhibiting thyroid dysfunction were also smokers. However, this possible association between PPP and thyroid dysfunction could be attributed to tobacco use rather than the presence of PPP [3]. Tobacco use can induce vasoconstriction or cause immune system dysfunctions, such as functional and morphological alterations in the polymorphonuclear leucocytes and an exaggerated release of chemotactic factors [12, 18]. Moreover, giving up the habit does not generally lead to the disappearance of PPP [19], which explains the significance of immune dysregulation. However, we did not find any correlation between the autoantibodies and tobacco use.

Type 2 diabetes mellitus is more frequent among women (23-76 years old, mean age 55 years) with PPP in Sweden [5]. In addition, an increased predisposition toward diabetes in PPP has been discussed by Uehara [20]. An abnormal glucose tolerance test was found in $55(66.3 \%)$ of 83 patients with PPP, and the deranged carbohydrate metabolism may be partly responsible for the increased susceptibility to focal infections in patients with PPP [20]. Several pathogenic factors, such as the serum calcium, tobacco use and obesity, which may predispose patients to type 2 diabetes should be taken into consideration, but these variables are not enough to explain the excess risk of diabetes in patients with PPP [5].

The IR is a subnormal biological response to normal insulin concentrations. The IR and relative insulin deficiency, with complex interactions among many genes and environmental factors, are the two of the major causes of type 2 diabetes mellitus [21-23]. The fasting insulin concentration is a useful marker to identify those subjects with IR, and the optimal cut-off point has been identified as $15.7 \mathrm{mU} / \mathrm{l}$ (109 pmol/l) for insulin [24]. In another study, the $75^{\text {th }}$ and $90^{\text {th }}$ percentiles defined as the cut-off values for IR and HOMA were 12-17.2 $\mathrm{mU} / \mathrm{I}$ and $2.6-4.1 \mathrm{\mu U} / \mathrm{ml}$, respectively. However, IR has not been previously studied in PPP patients. Although the fasting blood glucose, fasting insulin and HOMA index in the patients with PPP were slightly higher than in the controls, the fasting blood glucose, insulin cut-off level $(12 \mathrm{mU} / \mathrm{l})$ and HOMA index were not found to be useful in the evaluation of PPP. No correlations were found in these parameters between the controls and the PPP patients. Moreover, these parameters were not effective or predictive in the uni/multivariate analyses $(p>0.05)$.

Tobacco use and female gender have been shown to increase the risk of PPP in several other studies. Correspondingly, female gender and smoking were the most important risk factors for PPP according to our study. Based on the results of previous studies, the anti-TPO rate is normally $8.4 \%$ in the general population, but it is $22.5 \%$ in patients with PPP. In our study, the rates of thyroid pathologies and autoantibodies were $60 \%$ and $42 \%$, respectively, which could be associated with the high antiTPO levels in the female gender. Although there have been study results regarding the increased risk of type 2 dia- 
betes and impaired carbohydrate metabolism, IR has not been previously studied in PPP patients. In our research, no correlation was detected between IR and PPP; however, additional studies are necessary to clarify the relationships between PPP, thyroid disease and diabetes mellitus.

\section{Conflict of interest}

The authors declare no conflict of interest.

\section{References}

1. Mrowietz U, van de Kerkhof PC. Management of palmoplantar pustulosis: do we need to change? Br J Dermatol 2011; 164: 942-6.

2. Brunasso AM, Puntoni M, Aberer W, et al. Clinical and epidemiological comparison of patients affected by palmoplantar plaque psoriasis and palmoplantar pustulosis: a case series study. Br J Dermatol 2013; 168: 1243-51.

3. Gimenez-Garcia R, Sanchez-Ramon S, Cuellar-Olmedo LA. Palmoplantar pustulosis: a clinicoepidemiological study. The relationship between tobacco use and thyroid function. J Eur Acad Dermatol Venereol 2003; 17: 276-9.

4. Douroudis K, Kingo K, Traks T, et al. ATG16L1 gene polymorphisms are associated with palmoplantar pustulosis. Hum Immunol 2011; 72: 613-5.

5. Hagforsen E, Michaelsson K, Lundgren E, et al. Women with palmoplantar pustulosis have disturbed calcium homeostasis and a high prevalence of diabetes mellitus and psychiatric disorders: a case-control study. Acta Derm Venereol 2005; 85: 225-32.

6. Michaelsson G, Kristjansson G, Pihl Lundin I, Hagforsen E. Palmoplantar pustulosis and gluten sensitivity: a study of serum antibodies against gliadin and tissue transglutaminase, the duodenal mucosa and effects of gluten-free diet. Br J Dermatol 2007; 156: 659-66.

7. Matthews DR, Hosker JP, Rudenski AS, et al. Homeostasis model assessment: insulin resistance and beta-cell function from fasting plasma glucose and insulin concentrations in man. Diabetologia 1985; 28: 412-9.

8. Jurik AG, Ternowitz T. Frequency of skeletal disease, arthroosteitis, in patients with pustulosis palmoplantaris. J Am Acad Dermatol 1988; 18: 666-71.

9. Hellgren L, Mobacken H. Pustulosis palmaris et plantaris. Prevalence, clinical observations and prognosis. Acta Derm Venereol 1971; 51: 284-8.

10. Christopher E. Pustular eruptions of palms and soles. In: Dermatology in General Medicine. Fitzpatrick TB, Eisen AZ, Woloff K, et al. (eds). McGraw-Hill, New York 1993; 648-54.

11. Eriksson MO, Hagforsen E, Lundin IP, Michaelsson G. Palmoplantar pustulosis: a clinical and immunohistological study. Br J Dermatol 1998; 138: 390-8.

12. Akiyama T, Seishima M, Watanabe $H$, et al. The relationships of onset and exacerbation of pustulosis palmaris et plantaris to smoking and focal infections. J Dermatol 1995; 22: 930-4.

13. Rosen K, Lindstedt G, Mobacken H, Nystrom E. Thyroid function in patients with pustulosis palmoplantaris. J Am Acad Dermatol 1988; 19: 1009-16.

14. Enfors W, Molin L. Pustulosis palmaris etplantaris. A followup study of a ten-year material. Acta Derm Venereol 1971; 51: 289-94.
15. Rosen K, Mobacken H, Nilsson LA. Increased prevalence of antithyroid antibodies and thyroid diseases in pustulosis palmoplantaris. Acta Derm Venereol 1981; 61: 237-40.

16. Tunbridge WM, Evered DC, Hall R, et al. The spectrum of thyroid disease in a community: the Whickham survey. Clin Endocrinol (Oxf) 1977; 7: 481-93.

17. Gul U, Cakmak S, Gonul M, et al. Should thyroid function tests and thyroid autoantibodies be investigated in chronic urticaria? Asthma Allergy Immunol 2009; 7: 39-43.

18. Camp RDR. Psoriasis. In: Texbook of Dermatology. Champion RH, Burton JL, Burns DA (eds.). Blackwell Science, Oxford 1998; 1634-5.

19. O'Doherty CJ, Maclntyre C. Palmoplantar pustulosis and smoking. Br Med J (Clin Res Ed) 1985; 291: 861-4.

20. Uehara M. Pustulosis palmaris et plantaris. A review of clinical features and aggravating factors. Acta Otolaryngol Suppl. 1983; 401: 7-11.

21. Beck-Nielsen H, Groop LC. Metabolic and genetic characterization of prediabetic states. Sequence of events leading to non-insulin-dependent diabetes mellitus. J Clin Invest 1994; 94: 1714-21.

22. Robertson RP. Antagonist: diabetes and insulin resistancephilosophy, science, and the multiplier hypothesis. J Lab Clin Med 1995; 125: 560-4.

23. Kahn CR. Banting lecture. Insulin action, diabetogenes, and the cause of type II diabetes. Diabetes 1994; 43: 1066-84.

24. McLaughlin T, Abbasi F, Cheal K, et al. Use of metabolic markers to identify overweight individuals who are insulin resistant. Ann Intern Med 2003; 139: 802-9. 